\title{
Molecular Dynamics Simulations of Large Integral Membrane Proteins with an Implicit Membrane Model
}

\author{
Seiichiro Tanizaki ${ }^{\dagger}$ and Michael Feig*,†, \\ Department of Biochemistry and Molecular Biology and Department of Chemistry, Michigan State University, \\ East Lansing, Michigan 48824-1319
}

Received: August 19, 2005; In Final Form: November 4, 2005

\begin{abstract}
The heterogeneous dielectric generalized Born (HDGB) methodology is an the extension of the GBMV model for the simulation of integral membrane proteins with an implicit membrane environment. Three large integral membrane proteins, the bacteriorhodopsin monomer and trimer and the BtuCD protein, were simulated with the HDGB model in order to evaluate how well thermodynamic and dynamic properties are reproduced. Effects of the truncation of electrostatic interactions were examined. For all proteins, the HDGB model was able to generate stable trajectories that remained close to the starting experimental structures, in excellent agreement with explicit membrane simulations. Dynamic properties evaluated through a comparison of B-factors are also in good agreement with experiment and explicit membrane simulations. However, overall flexibility was slightly underestimated with the HDGB model unless a very large electrostatic cutoff is employed. Results with the HDGB model are further compared with equivalent simulations in implicit aqueous solvent, demonstrating that the membrane environment leads to more realistic simulations.
\end{abstract}

\section{Introduction}

Membrane proteins encompass a large and important class of proteins. Therefore, a molecular understanding of their structure, dynamics, and function is fundamental in the biological sciences. ${ }^{1-3}$ Nevertheless, membrane proteins are relatively poorly understood, because experimental studies of membrane environments remain very challenging. ${ }^{4,5}$ As a complimentary approach to experiments, computer simulations can provide insight about atomic-scale dynamics and functional mechanisms of biological molecules in general and membrane proteins in particular. ${ }^{6-8}$ All-atom representations of the surrounding biomolecular environment (water, lipids, ions, etc.) are commonly employed in order to provide the most detailed description of solute-solvent interactions..$^{9-11}$ However, a fully explicit representation of the environment introduces significant additional computational cost ${ }^{12-14}$ and, in the case of heterogeneous membrane environments, also a significant level of system complexity. ${ }^{15}$

As an alternative to a fully explicit description of the environment, mean field approaches subsume the solvent degrees of freedom into an implicit formulation that captures the average effect of the solvent rather than instantaneous interactions with explicit solvent molecules. ${ }^{12-14}$ Molecular environments are often approximated as a dielectric continuum, where the electrostatic solvation energy due to polarization at a dielectric interface is described by the Poisson-Boltzmann (PB) equation. Although the PB equation may be solved numerically and can be applied to heterogeneous environments, ${ }^{16-20}$ it is generally difficult to balance efficiency and

* To whom correspondence should be addressed: feig@msu.edu.

$\dagger$ Department of Biochemistry and Molecular Biology.

$\div$ Department of Chemistry. accuracy in direct solutions to the $\mathrm{PB}$ equation for the purpose of molecular dynamics simulations. ${ }^{21-24}$

The generalized Born (GB) model offers an efficient analytical approximation to the electrostatic solvation free energy of a solute in aqueous solution according to PB theory. ${ }^{25}$ A number of GB variants have been proposed in recent years that are summarized in a recent review. ${ }^{12}$ The accuracy of modern GB implementations can approximate the electrostatic component of the solvation free energy within a relative error of $0.5-1 \%$ compared to reference numerical solutions of the Poisson equation. ${ }^{24,26}$ However, the standard GB formalism is limited to homogeneous environments, while implicit models of membrane environments require a heterogeneous model with a low dielectric lipid interior that is surrounded by high dielectric lipid headgroups and waters. ${ }^{27}$ Recently, the GB formalism has been extended by us $^{28}$ and others ${ }^{29,30}$ in order to allow the implicit modeling of membrane environments.

In first implementations of an implicit membrane model within the generalized Born formalism, the solute cavity was extended into the hydrophobic region of the phospholipid tails. The result is in effect still a two-dielectric model with $\epsilon=1$ for the solute and lipid region surrounded by $\epsilon \approx 80$ under the assumption that the electric field of the protein does not elicit a significant dielectric response from the lipid tail region. In a first implementation of this idea, Spassov et al. extended a linear version of the asymptotic pairwise GB approach that was introduced originally by Qui et al. ${ }^{31}$ for modeling membrane environments (called GBSA/IM) ${ }^{30}$ The method was tested with nanosecond molecular dynamics simulations of the fusion peptide influenza virus hemagglutinin HA2 peptide (20 residues). Following the same idea of extending the solute cavity into the lipid region, Im et al. implemented a two-dielectric membrane model, called GBIM, based on GBSW (GB with simple switching function). ${ }^{32}$ The GBIM model has been 
employed in simulations of bee venom melittin (26 residues), the transmembrane domain of the M2 protein from Influenza A virus (25 residues), and the glycophorin A monomer (25 residues) and dimer (50 residues). ${ }^{29}$ Furthermore, the GBIM model was used for folding simulations of the major $\mathrm{pVIII}$ coat protein from filamentous bacteriophage fd (50 residues $)^{33}$ and other synthetic small peptides. ${ }^{34}$

Most recently, we have proposed the extension of the GBMV (GB with molecular volume) method ${ }^{26}$ to an implicit membrane model called HDGB. ${ }^{28}$ In contrast to the previously described approaches, the HDGB model allows multiple layers of different dielectric constants. In particular, the dielectric constants of the protein interior and the hydrophobic interior of the lipid bilayer need not be equal in the HDGB model, which allows for a polarization response at the protein/lipid interface. This model was tested first with molecular dynamics simulations of bee venom melittin and the bacteriorhodopsin monomer from Halobacterium salinarum. ${ }^{28}$

It should be noted that outside the GB methodology the effective energy function IMM1 is also available for simulating a protein in an implicit membrane environment. ${ }^{35,36}$ IMM1 is an extension of the empirical solvent-exclusion model EEF1. ${ }^{37}$ The IMM1 model is based on the transfer free energies from vapor phase to cyclohexane, and the membrane is simply viewed as a nonpolar hydrophobic homogeneous medium. Essentially, the IMM1 model also represents the membrane as a two-layer system. Beyond a short simulation of bacteriorhodopsin over $200 \mathrm{ps},{ }^{35}$ the IMM1 has recently added the ability to simulate the anionic lipid membrane environment, and the model has been tested on simulations of membrane-binding peptides. $^{36}$

One of the main motivations for using an implicit membrane model instead of an explicit membrane representation lies in the possibility for extending system sizes and simulation times beyond what is currently practical with explicit membrane simulations. Therefore, it is important to demonstrate that implicit membrane models can be applied in the simulation of large membrane-interacting proteins. In this study, we are reporting long-time molecular dynamics simulations of the bacteriorhodopsin monomer and trimer as well as the 1110 residue BtuCD protein with the HDGB implicit membrane model.

In the next section, the underlying theory for the heterogeneous dielectric generalized Born formalism is described briefly along with a description of the simulation methods employed in this study, followed by a presentation and discussion of results.

\section{Methods}

HDGB Model. While the details of the HDGB model have been described elsewhere, ${ }^{28}$ the relevant features are summarized here. In general, the solvation free energy of a biomolecule in a given environment can be decomposed into the sum of the electrostatic contribution $\Delta G_{\text {elst }}$ and the nonpolar contribution $\Delta G_{\mathrm{np}}{ }^{38}$

$$
\Delta G_{\mathrm{sol}}=\Delta G_{\mathrm{elst}}+\Delta G_{\mathrm{np}}
$$

In a simple homogeneous environment, the electrostatic contribution can be obtained according to the generalized Born expression proposed by Still et al. ${ }^{25}$ In the HDGB model, the following modified expression is employed in order to
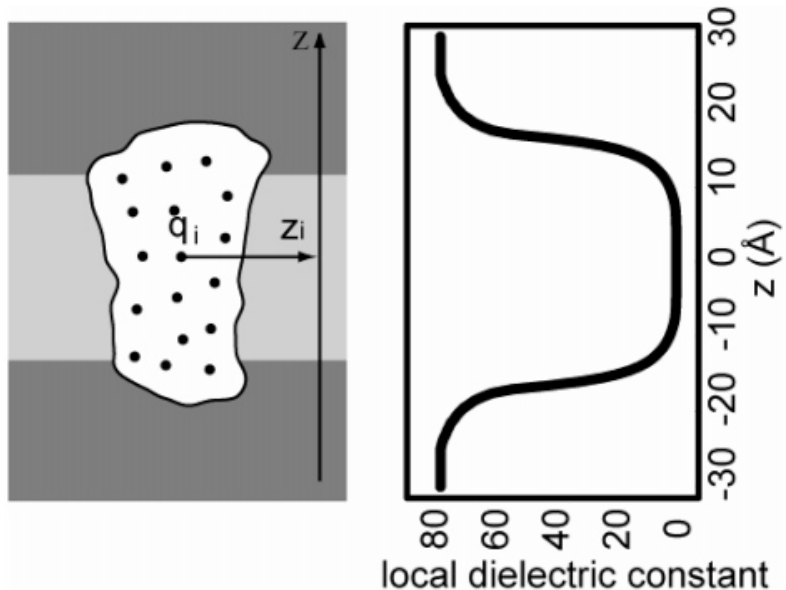

Figure 1. Schematics of a heterogeneous implicit membrane environment (left); local dielectric constant along the direction normal to the implicit membrane plane (right).

incorporate a heterogeneous dielectric environment

$$
\begin{aligned}
\Delta G_{\mathrm{elst}}=-166 \sum_{i=1}^{n} \sum_{j=1}^{n}\left[1-\frac{1}{(1 / 2)\left(\epsilon_{i}+\epsilon_{j}\right)}\right] & \frac{q_{i} q_{j}}{\sqrt{r_{i j}{ }^{2}+\alpha_{i}\left(\epsilon_{i}\right) \alpha_{j}\left(\epsilon_{j}\right) \exp \left[-r_{i j}{ }^{2} / F \alpha_{i}\left(\epsilon_{i}\right) \alpha_{j}\left(\epsilon_{j}\right)\right]}}
\end{aligned}
$$

where $n$ is the number of atoms, $q$ is the atomic charge in electron units, $r$ is the interatomic distance in angstroms, and $F$ is a dimensionless empirical parameter taken to be 8 . In the HDGB model, an effective dielectric constant is assigned to each atom according to its local environment. In the case of the membrane, the effective dielectric constant varies simply as a function of $z$, the distance from the membrane center. The dielectric profile is obtained by solving the Poisson equation for a spherical probe ion that is translated across the discrete dielectric layers describing the membrane environment. ${ }^{28}$ The resulting dielectric profile $\epsilon(z)$ for dipalmitoylphosphatidylcholine (DPPC) bilayers is shown in Figure 1. The HDGB model is based on the GBMV model, ${ }^{26}$ a generalized Born (GB) version that utilizes the standard molecular volume and can predict the electrostatic solvation free energy very accurately when compared with Poisson theory. ${ }^{26}$

In the GBMV formalism, the effective Born radius $\alpha$ is determined according to the Coulomb Field approximation including a correction term. ${ }^{26}$ For varying dielectric environments, the Born radii $\alpha(\epsilon)$ are calculated as a function of the dielectric constant as proposed by Feig et al. ${ }^{39}$

$$
\alpha(\epsilon)=\frac{1}{C_{0} A_{4}+C_{1}\left(\frac{3 \epsilon}{3 \epsilon+2}\right) A_{7}}+D+\frac{E}{\epsilon+1}
$$

where

$$
\begin{gathered}
A_{4}=\frac{1}{R_{i}}-\frac{1}{4 \pi} \int_{\text {solute }, r>R_{i}} \frac{1}{r^{4}} \mathrm{~d} V \\
A_{7}=\left(\frac{1}{4 R_{i}^{4}}-\frac{1}{4 \pi} \int_{\text {solute }, r>R_{i}} \frac{1}{r^{7}} \mathrm{~d} V\right)^{1 / 4}
\end{gathered}
$$

The parameters $C_{0}, C_{1}, D$, and $E$ are set to be $0.3225,1.085$, -0.14 , and $-0.15 .^{39}$ The volume integration is performed over 
the solute volume except for a spherical exclusion region of radius $R_{i}$ centered at the $i$ th atom in order to avoid the numerical singularity at $r=0$.

The nonpolar contribution is approximated by the solventaccessible surface area (SASA) model

$$
\Delta G_{\mathrm{np}}=\gamma \sum_{i=1}^{n} S\left(z_{i}\right) A_{i}
$$

where $A_{i}$ is a solvent-accessible surface area of the $i$ th atom, $z_{i}$ is a position of the $i$ th atom along the normal direction, and $\gamma$ is an empirical surface tension parameter set to $0.015 \mathrm{kcal} / \mathrm{mol}$ on the basis of results from melittin test simulations. ${ }^{28}$ The change in surface tension from lipid to bulk water is modeled with the switching function $S(z)$. The shape of $S(z)$ is taken from simulation results for the insertion of molecular oxygen into lipid bilayers ${ }^{40}$ and fitted to the following functional form

$$
\left[\begin{array}{ll}
S(z)= & \left(0 \leq|z|<z_{b}\right) \\
c \frac{\left(|z|-z_{a}\right)^{2}\left(3 z_{b}-2|z|-z_{a}\right)}{\left(z_{b}-z_{a}\right)^{3}} & \left(z_{b} \leq|z|<z_{c}\right) \\
(1-c) \frac{\left(|z|^{2}-z_{b}^{2}\right)^{2}\left(3 z_{c}{ }^{2}-2|z|^{2}-z_{b}^{2}\right)}{\left(z_{c}^{2}-z_{b}^{2}\right)^{3}} & \text { (otherwise) }
\end{array}\right]
$$

with $c, z_{a}, z_{b}$, and $z_{c}$ determined as $0.32,0.5,9.2$, and $25 \AA$, respectively.

The HDGB method is available in the macromolecular simulation package CHARMM version c33a1. ${ }^{41,42}$

Test Systems. To examine the quality of molecular dynamics simulations with the HDBG model, simulations of bacteriorhodopsin (bR) monomer (228 residues), bacteriorhodoposin trimer (684 residues), and the BtuCD dimer (1110 residues) were carried out over $10 \mathrm{~ns}, 5 \mathrm{~ns}$, and $5 \mathrm{~ns}$, respectively. Bacteriorhodopsin from Halobacterium salinarum (Figure 2A) is an integral membrane protein that converts the energy of light into a proton gradient. ${ }^{1}$ Bacteriorhodopsin monomer consists of 7 transmembrane $\alpha$-helices A through $\mathrm{G}$ with a retinal linked to Lys 216 of helix $G$ and in vivo arranged into a hexagonal twodimensional crystal of homotrimers. ${ }^{43}$ Because bR has been studied extensively, both experimental and theoretical data from explicit lipid simulations of the monomeric and trimeric forms are available for comparison. The Escherichia coli BtuCD integral inner membrane protein (Figure 2B) is an ABC (ATPbinding cassette) transporter importing vitamin $\mathrm{B}_{12} .{ }^{44}$ The crystal structure of the BtuCD protein (Figure 2B) shows that the protein consists of 4 subunits. Homodimers are formed between the membrane-spanning domain BtuC and the cytoplasmic ATPbinding domain BtuD. ${ }^{45}$ As of this writing, only 1 simulation study was reported using a united-atom force field. ${ }^{46}$ In addition to simulations with the HDGB model, simulations of all 3 proteins were also performed in a homogeneous implicit aqueous environment using the standard GBMV model ${ }^{26}$ for comparison with the HDGB model.

\section{Simulation Methods}

The initial structures of $\mathrm{bR}$ and BtuCD were taken from X-ray crystallographic structures (PDB entries $1 \mathrm{QHJ}{ }^{47}$ and $1 \mathrm{~L} 7 \mathrm{~V},{ }^{45}$ respectively). Missing hydrogen atoms were added by using the HBUILD module in CHARMM ${ }^{41,42}$ Standard protonation states $(\mathrm{pH}=7)$ were assumed for all amino acids of bR except that the residues Asp96, Asp115, and Glu204 were protonated. ${ }^{48}$
(A)

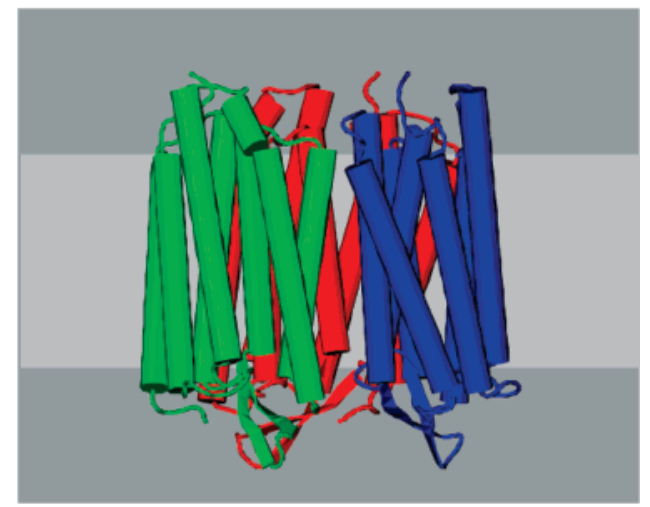

(B)

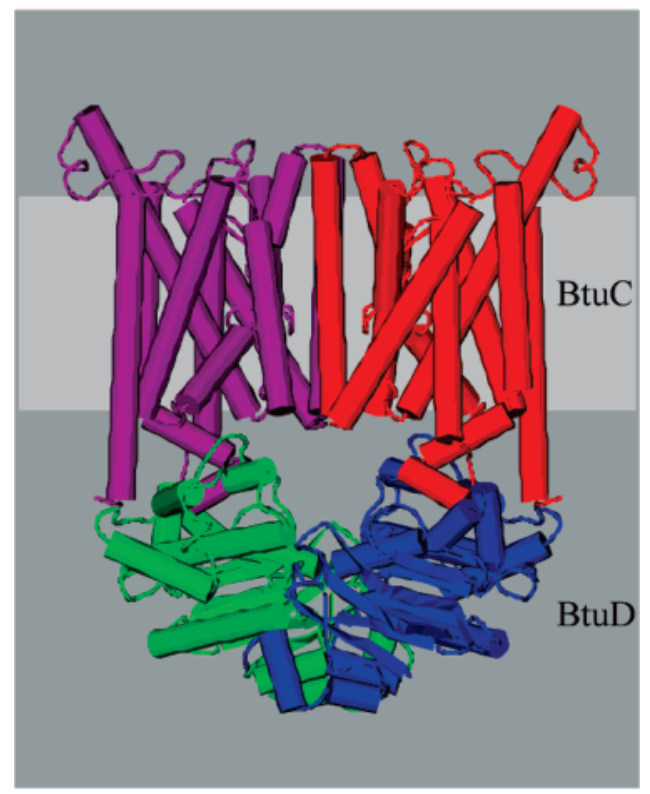

Figure 2. The initial configuration of the bacteriorhodopsin trimer (A, PDB ID 1QHJ ${ }^{47}$ ) and the BtuCD protein (B, PDB ID 1L7V ${ }^{45}$ ) in an implicit membrane environment. The figures were prepared by VMD. ${ }^{68}$

Standard protonation states were used for all amino acids of the BtuCD protein. Proteins were terminated with a charged terminus at both ends. For bR, the X-ray crystallographic structure includes the position of oxygen atoms of a number of water molecules. We included only the buried water molecules (nine water molecules per monomer). In all cases, the CHARMM22 all-atom force field parameters were employed ${ }^{42}$ with the CMAP $\varphi / \psi$ torsion potential correction. ${ }^{49}$ The force field parameters of the protonated Schiff base in bR is described in Saam et al..$^{50}$

The initial structures were energy-minimized in order to release the side-chain strains before beginning the simulations. The energy-minimized structure was then placed so that its principal axis coincided with the $z$-axis and its center of the mass was at the origin (Figure 2). The systems were heated slowly from 0 to $300 \mathrm{~K}$ over the course of $160 \mathrm{ps}$. The production phase was carried out at $300 \mathrm{~K}$. Langevin dynamics was used to control the temperature of the systems with a friction coefficient of $5 \mathrm{ps}^{-1}$ for all non-hydrogen atoms. The nonbonded interactions and the GB solvation term were switched off smoothly from $14 \AA$ to $16 \AA$ in the bR trimer and the BtuCD simulations. The bR monomer was simulated with cutoff 


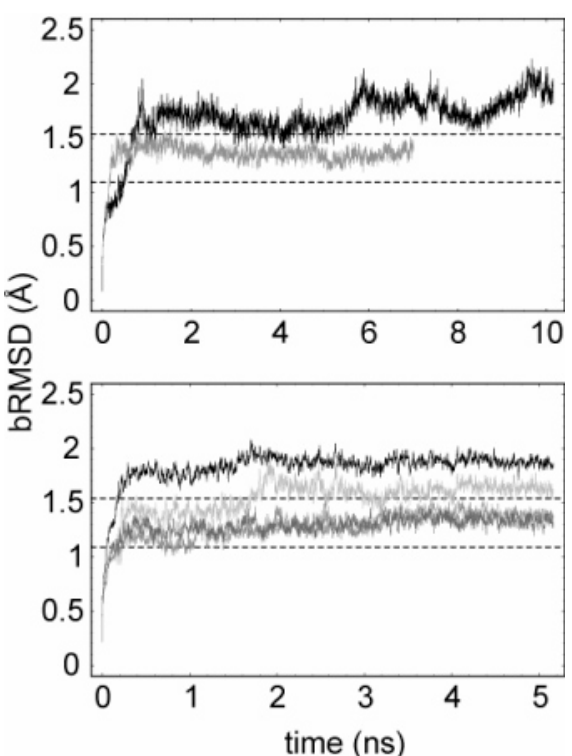

Figure 3. The backbone RMSD of the bacteriorhodopsin monomer (top) and trimer (bottom) from HDGB simulation. The dotted horizontal lines mark the range of bRMSD of the mean structures of the explicit trimer simulation. ${ }^{53}$ The bRMSD of each subunit is shown in gray, and the total bRMSD of the trimer is shown in black.

distances of $16 / 18 \AA$ as well as $36 / 38 \AA$. The bR monomer simulation with $16 / 18 \AA$ has been reported previously ${ }^{28}$ and is included here for comparison. The bond lengths involving hydrogen atoms were fixed by using the SHAKE algorithm, ${ }^{51}$ so that a simulation time step of $2 \mathrm{fs}$ could be used. Coordinates were saved every $2 \mathrm{ps}$, and average values were computed over the last $3 \mathrm{~ns}$ of simulation time for the bR trimer and BtuCD and over the last $5 \mathrm{~ns}$ for the bR monomer. Tilt angles of bR helices were computed with the algorithm proposed by Åqvist. ${ }^{52}$ All of the simulations were carried out with CHARMM, ${ }^{41,42}$ version c31b1, where the HDGB method was first implemented.

It took about 25 days to run $1 \mathrm{~ns}$ of the HDGB simulations of the bR trimer and the BtuCD dimer on 2 and 4 Xeon 3.06 $\mathrm{GHz}$ CPUs, respectively. The simulations of the $\mathrm{bR}$ monomer took 12 days on 2 CPUs per $1 \mathrm{~ns}$.

\section{Results}

In the following, results of HDGB molecular dynamics simulations of bacteriorhodopsin (monomer and trimer) and the BtuCD dimer are presented. The simulations are analyzed with respect to thermodynamic stability and dynamic properties in comparison with experiments and data from explicit membrane simulations. Furthermore, the effect of nonbonded truncation is examined for the bR monomer, and the results with the HDGB model are compared with simulations in implicit aqueous solvent.

Structural Stability. Figure 3 shows the backbone rootmean-square deviations (bRMSD) of bacteriorhodopsin monomer (top) and trimer (bottom) in multi-nanosecond molecular dynamics simulations with the HDGB implicit membrane model. Figure 5 shows the bRMSD of the BtuCD dimer over the course of $5 \mathrm{~ns}$. To calculate bRMSD values, all trajectories were oriented with respect to the $\mathrm{C}_{\alpha}$ atom of the respective experimental structures. In the case of the bR trimer and the BtuCD dimer, we monitored the total bRMSD with respect to the $\mathrm{C}_{\alpha}$ atoms of the experimental trimer and dimer structure relatively (black lines in Figure 3 and Figure 5) as well as the bRMSD of each $\mathrm{bR}$ monomer in the $\mathrm{bR}$ trimer and the $\mathrm{BtuC}$ and $\mathrm{BtuD}$ domains. Overall, the structures of the bR monomer, bR trimer,
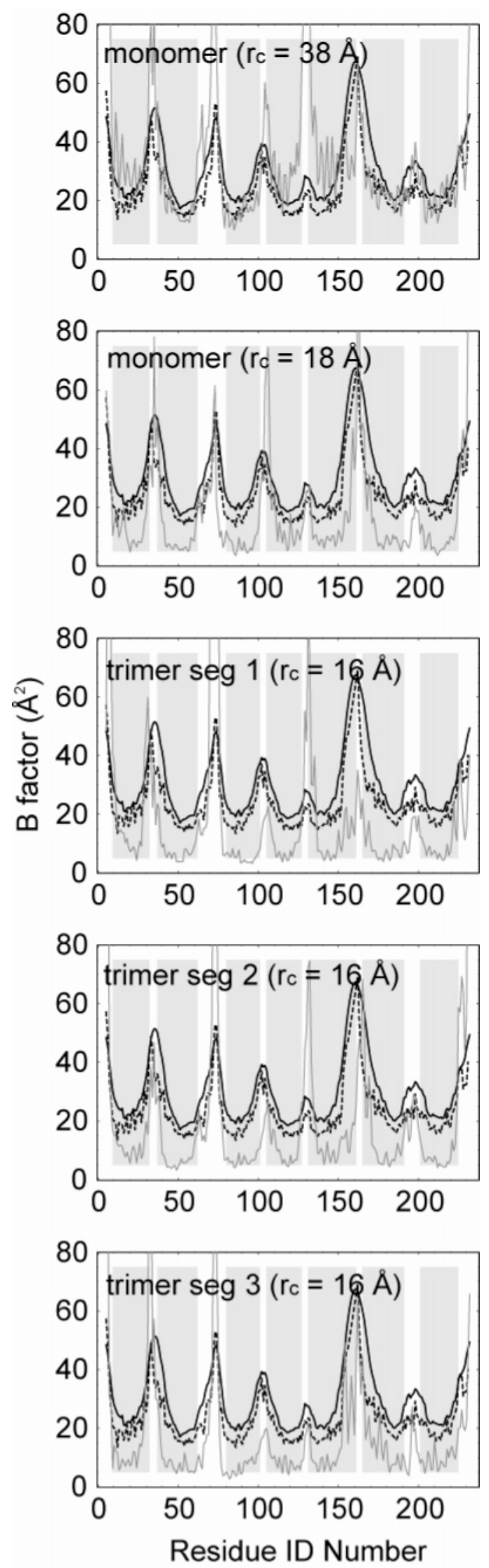

Figure 4. The B-factors of the $\mathrm{C}_{\alpha}$ atoms were calculated from their root-mean-square fluctuations. The solid gray line is from the HDGB simulation. The black solid (resolution $1.9 \AA)^{47}$ and dashed (resolution $1.55 \AA)^{22}$ lines are from the experiment. The region of the secondary structure elements (helices $A-G$ from the left) are indicated by the gray background. 


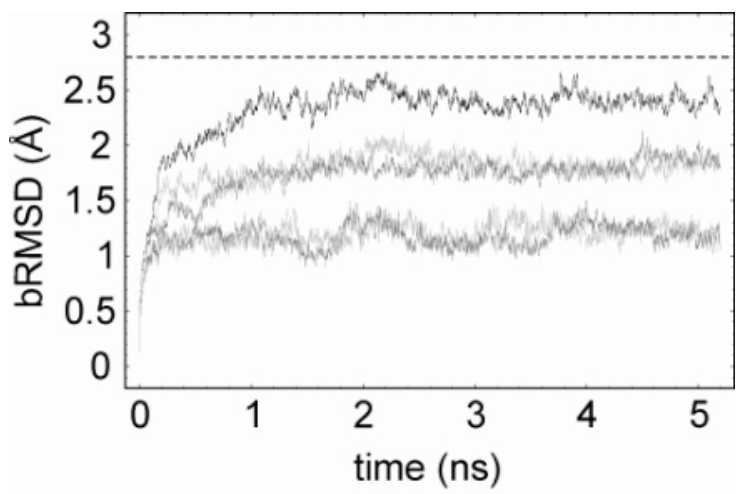

Figure 5. The backbone RMSD of the BtuCD from the HDGB simulation. Transmembrane subunit (BtuC) in gray color (top two), the nucleotide-binding domain (BtuD) in gray color (bottom two), and the total bRMSD in black.

TABLE 1: Comparison of Helix Tilt Angles (in deg) for Bacteriorhodopsin Measured from the X-ray Crystallographic Data (experiment) ${ }^{47}$ the HDGB Simulations (HDGB), and the Explicit Membrane Simulations (explicit) ${ }^{15 a}$

\begin{tabular}{crrrrr}
\hline helix & $\begin{array}{c}\text { experiment } \\
\text { trimer }\end{array}$ & $\begin{array}{c}\text { HDGB MD } \\
\text { trimer }\end{array}$ & $\begin{array}{c}\text { experiment } \\
\text { monomer }\end{array}$ & $\begin{array}{c}\text { HDGB MD } \\
\text { monomer }\end{array}$ & $\begin{array}{c}\text { explicit MD } \\
\text { monomer }\end{array}$ \\
\hline $\mathrm{A}$ & 23 & $24(13-32)$ & 17 & $17(12-22)$ & $18(13-29)$ \\
$\mathrm{B}$ & 5 & $8(2-16)$ & 13 & $7(3-11)$ & $14(5-22)$ \\
$\mathrm{C}$ & 11 & $13(5-22)$ & 19 & $14(8-19)$ & $15(7-23)$ \\
$\mathrm{D}$ & 8 & $10(3-17)$ & 14 & $12(7-17)$ & $16(0-19)$ \\
$\mathrm{E}$ & 11 & $16(6-25)$ & 7 & $10(2-17)$ & $13(5-23)$ \\
$\mathrm{F}$ & 14 & $13(4-22)$ & 4 & $7(1-12)$ & $3(0-14)$ \\
$\mathrm{G}$ & 16 & $15(2-24)$ & 8 & $8(3-12)$ & $5(1-18)$
\end{tabular}

a Average tilt angles are given for simulation data with minimum, and maximum tilt angles are in parentheses.

and BtuCD dimer were stable and remained close to the starting experimental structure over the entire simulation time. The bRMSD of the mean structure from the bR monomer simulation trajectory was $1.69 \AA$ ( $38 \AA$ cutoff), and the bRMSD of the mean structure of each $\mathrm{bR}$ monomer from the trimer simulation trajectories were $1.53,1.23$, and $1.25 \AA$. Our results of the monomer and the trimer are very similar to the range of bRMSD of the mean structures $(1.09-1.54 \AA$ ) found from a recent $5 \mathrm{~ns}$ explicit membrane simulation of the bR trimer. ${ }^{53}$ In the simulation of the BtuCD dimer, a total bRMSD of $2.30 \AA$ was obtained, while the bRMSDs of the mean structures of the BtuC subunits were 1.77 and $1.72 \AA$ and those of BtuD subunits were 1.05 and $0.97 \AA$. These results indicate that the transmembrane domain deviates more from the experimental structure than the cytoplasmic nucleotide-binding domain, but the results are overall in close agreement with a recent explicit membrane simulation of BtuCD, ${ }^{46}$ where a total RMSD of $\sim 2.8 \AA$ was reached after $1 \mathrm{~ns}$ and maintained over a total simulation time of $15 \mathrm{~ns}$ for the entire BtuCD dimer (E. Oloo, personal communication).

To evaluate the quality of the sampled structures further, the bR helix tilt angles (relative to the direction normal to the plane of membrane) were compared with experiment and explicit membrane simulation data. Average, minimum, and maximum tilt angles of each helix of the bR monomer (from the $38 \AA$ cutoff simulation) and the trimer were calculated from the HDGB simulations and are shown in Table 1. In the case of the trimer, the tilt angles are averaged over all three segments. Tilt angles from the experimental structure were computed by orienting the principal axis of the trimer along the $z$-axis, perpendicular to the membrane bilayer. Very good agreement

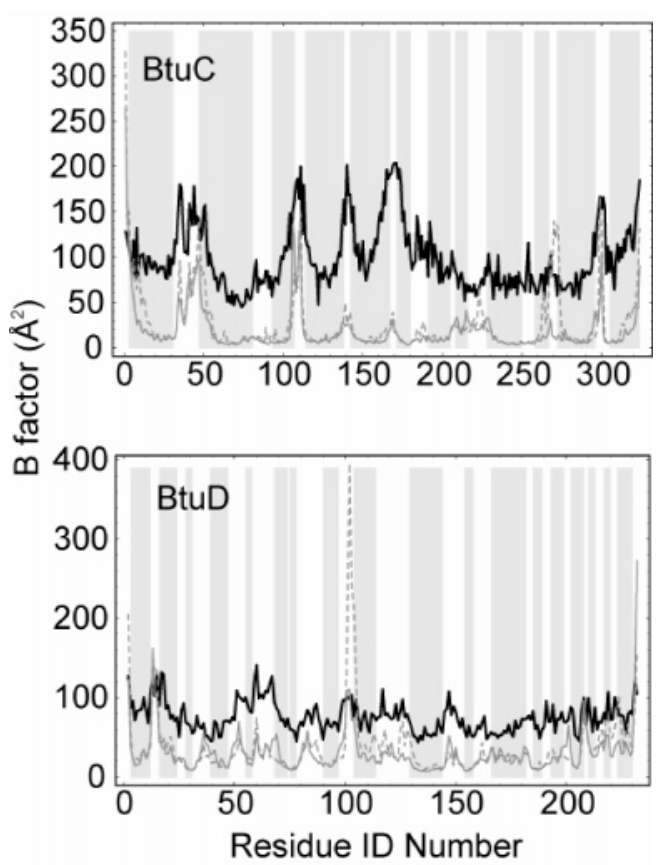

Figure 6. The B-factors of the $\mathrm{C}_{\alpha}$ atoms were calculated from their root-mean-square fluctuations. The dotted gray line is for the calculated values, and the dark solid line is for the experiment. ${ }^{45}$ The region of the secondary structure elements are indicated by the gray background.

is found between the average tilt angles from X-ray crystallography and the HDGB simulations of the trimer. The simulated tilt angles of the bR monomer are slightly different, especially for helices A, F, and G. The results from the HDGB match explicit membrane simulations of a bR monomer. ${ }^{15}$ They also match the experimental data if only the monomer is oriented along the $z$-axis. This finding may suggest that the $\mathrm{bR}$ monomer and bR trimer are oriented at a slightly different angles within the membrane bilayer. The minimum and maximum tilt angles indicate substantial fluctuations around the average values in the HDGB simulations, also in good agreement with the explicit membrane simulations. ${ }^{15}$

Effect of Nonbonded Cutoff. Although a nontruncated calculation of all nonbonded pair interactions is feasible in molecular dynamics simulations of small peptides in implicit solvent, practical limitations require a nonbonded cutoff, as the system size increases even without explicit representation of the environment. Commonly, nonbonded cutoff distances of 16$20 \AA$ are considered sufficient to avoid artifacts due to truncation of the Coulomb and electrostatic solvation energies. ${ }^{54}$ While most of the simulations reported here were stable with a $16 \AA$ cutoff, we did encounter difficulties with a simulation of the bacteriorhodopsin monomer when a $16 \AA$ cutoff was applied to the nonbonded interactions. In this case, the protein flipped from the expected vertical orientation to a horizontal orientation soon after the equilibration period, and it remained in the horizontal orientation (Figure 7) over the course of 9 ns until the simulation was stopped. Despite the unphysical orientation in the membrane, the structure surprisingly remained fully intact with a relatively low RMSD of $\sim 2.4 \AA$ after superposition with the experimental structure.

To examine the nature of this simulation artifact, electrostatic energy components with different cutoffs were compared between the vertical and horizontal orientations. The electrostatic GB solvation energy computed by the HDGB model for different cutoff distances is shown in Figure 8. The solvation energy fluctuates wildly for cutoff distances of less than $20 \AA$, 

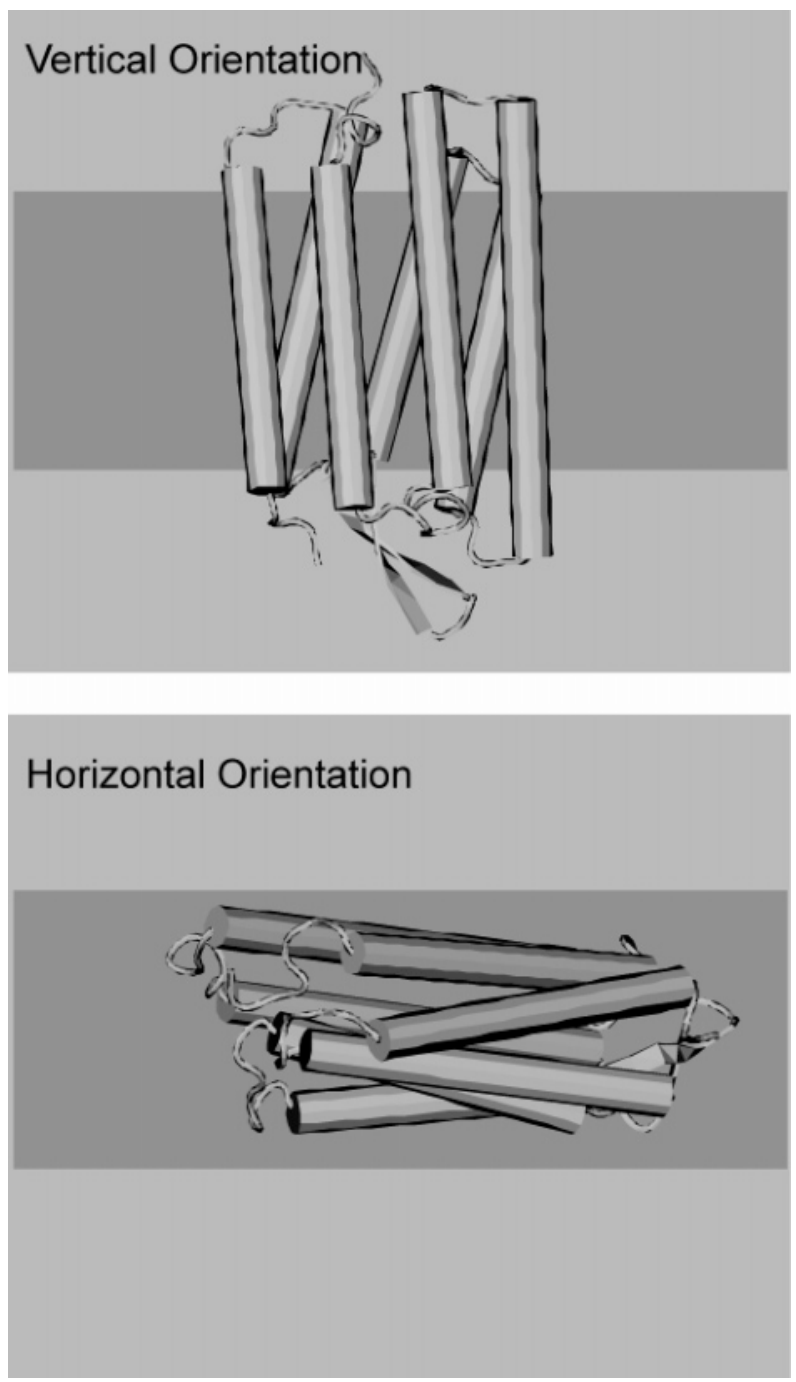

Figure 7. The HDGB simulation of the bacteriorhodopsin monomer with the cutoff distance of $16 \AA$ was started from the vertical orientation (top) and switched to the horizontal orientation (bottom) after equilibration. The figures were prepared by VMD. ${ }^{68}$

but it reaches the value obtained without any cutoffs only at cutoff distances of $25 \AA$ or more. The total electrostatic energy, that is, the solvation energy plus the nonbonded electrostatic interactions, shown in Figure 9, fluctuates much less as a function of the cutoff distance because of compensatory effects between the Coulomb and solvation contributions. Nevertheless, large fluctuations are still visible for cutoff distances up to 20 $\AA$. More importantly, the relative energy between the vertical and horizontal orientations actually has the wrong sign at a cutoff distance of $16 \AA$, suggesting a stabilization of the horizontal orientation over the vertical orientation, which is in fact observed in the simulation. In contrast, simulations of the bR monomer with larger cutoff distances of $18 \AA$ and $38 \AA$ did not lead to rotation of the monomer.

Dynamic Properties. In addition to thermodynamic stability, it is equally important to examine dynamic properties of the simulated membrane. To address the dynamic aspect, B-factors of $\mathrm{C}_{\alpha}$ atoms were calculated from the HDGB simulations and compared with data from experiments $22,45,47$ and an explicit membrane simulation. ${ }^{53}$ Figures 4 and 6 show the results for the bR and BtuCD systems. Qualitatively, the B-factors obtained from the implicit simulations agree very well with the experimental data, nicely reproducing the alternation between rigid secondary structure elements and flexible loop regions. The

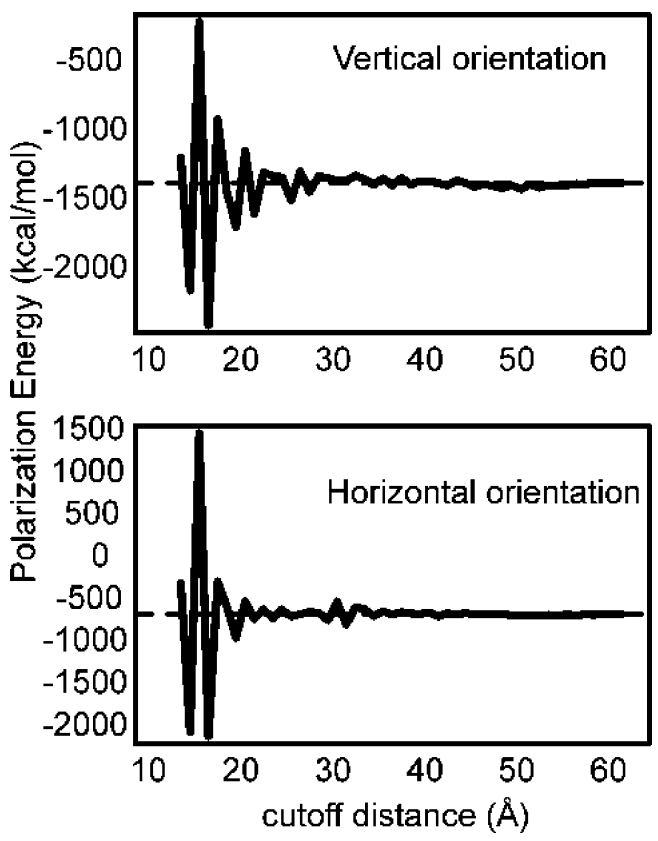

Figure 8. The effect of the cutoff distance on the GB polarization energy. The dotted line marks the energy computed without using the truncation method.
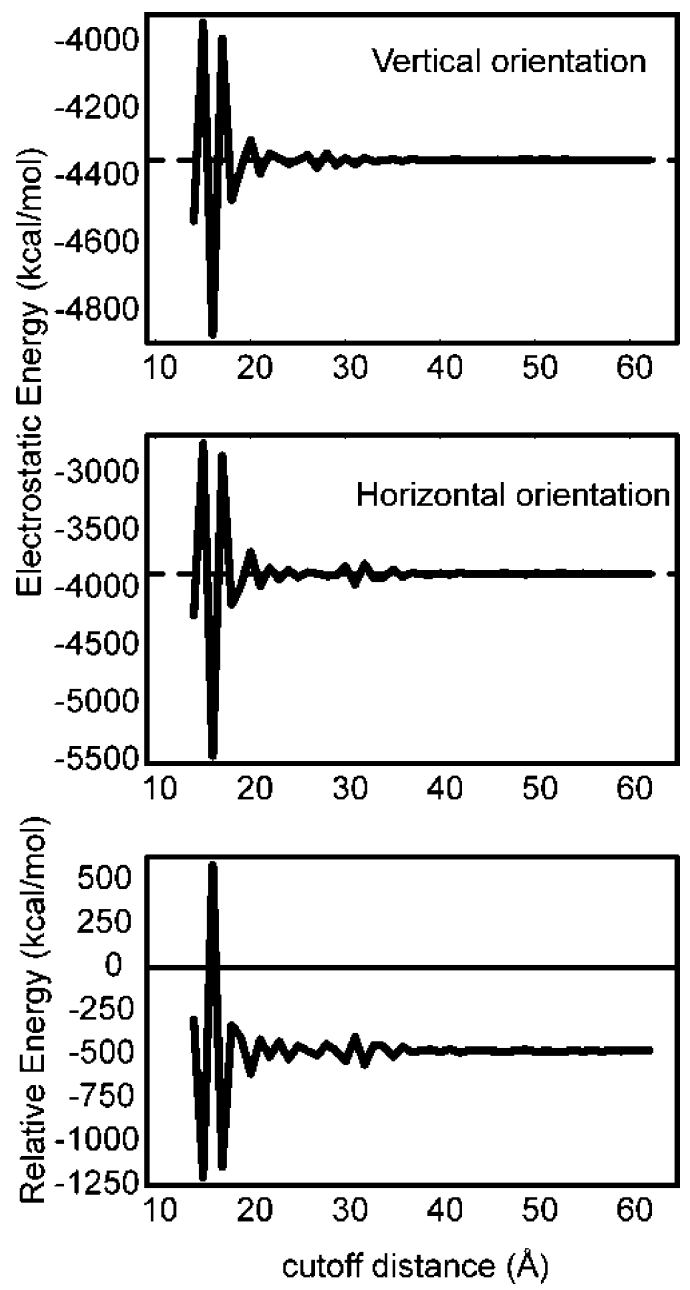

Figure 9. The effect of the cutoff distance on the total electrostatic energy. The dotted line marks the energy computed without using the truncation method.

quantitative agreement is also good, but in most cases, the baseline B-factors are somewhat lower than the experimental 


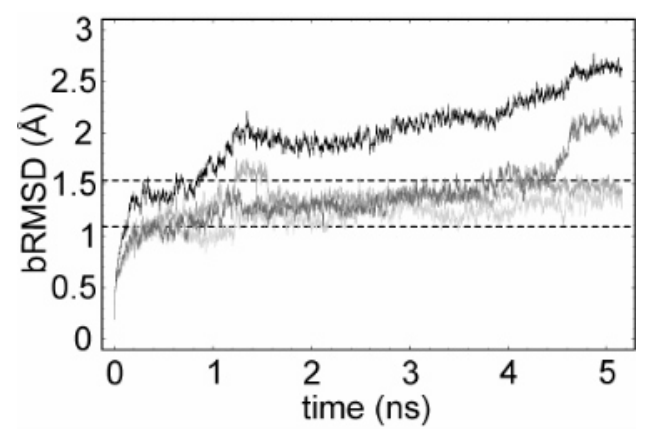

Figure 10. The backbone RMSD of the bacteriorhodopsin trimer from the implicit aqueous solvent simulation. The dotted horizontal lines mark the range of bRMSD of the mean structures of the explicit trimer simulation..$^{53}$ The bRMSD of each subunit is shown in gray color, and the total bRMSD of the trimer is shown in black.

values and also slightly lower than in the explicit membrane simulations, suggesting the possibility of slightly suppressed mobility in the implicit membrane simulations. However, it is particularly interesting that the B-factors calculated from the bR monomer simulation with a $38 \AA$ cutoff are closer, in fact very close, to the experimental numbers compared to the simulation of the same system simulated with an $18 \AA$ nonbonded cutoff distance.

In one loop region between residues 101-105 of one segment of $\mathrm{BtuD}$, the calculated values are substantially larger, suggesting extensive motion. This loop is located between two helices after the Q-loop of the BtuD and is completely exposed to the solvent. During the simulation, the loop undergoes a conformational transition, which explains the large B-factor values for this region in our simulation.

Effect of Implicit Membrane Environment. While all of the simulations described so far used the implicit membrane environment according to the HDGB model, another set of simulations of the same systems was run concurrently with an implicit aqueous environment. Among the three membrane proteins, the most significant difference was seen in the simulation of bacteriorhodopsin trimer (Figure 10). With an aqueous environment, the overall bRMSD steadily increases toward $\sim 2.6 \AA$ (vs $1.8 \AA$ with the HDGB model) indicating that the stability of the overall trimer structure in an aqueous environment is not maintained as well as in the membrane environment. Furthermore, the bRMSD of the third subunit increases from less than $1.5 \AA$ to about $2 \AA$ after 4 ns in the aqueous environment, while all subunits in the implicit membrane environment kept a stable bRMSD of less than $1.9 \AA$ throughout the $5 \mathrm{~ns}$ simulation. This finding suggests that the stability of the bR monomer is also compromised by the aqueous solvent environment. Other simulations of the bR monomer and the BtuCD protein did not show differences as significant in bRMSD between the two different environments (data not shown).

Figure 11 shows the B-factors of $\mathrm{C}_{\alpha}$ atoms calculated from the implicit aqueous solvent simulation. The qualitative agreement in the aqueous solvent simulation is not as good as in the HDGB model simulation. In particular, in the bR trimer, the first helix in the first and second segments and the fourth helix in the first and third segments have relatively large B-factors compared to those of other helices. However, the baseline B-factors are in slightly better agreement with experiment than with the HDGB model (Figure 4), indicating increased flexibility with the aqueous solvent environment. The qualitative features of B-factors of the transmembrane subunits BtuC in the BtuCD aqueous solvent simulations are notably different from the
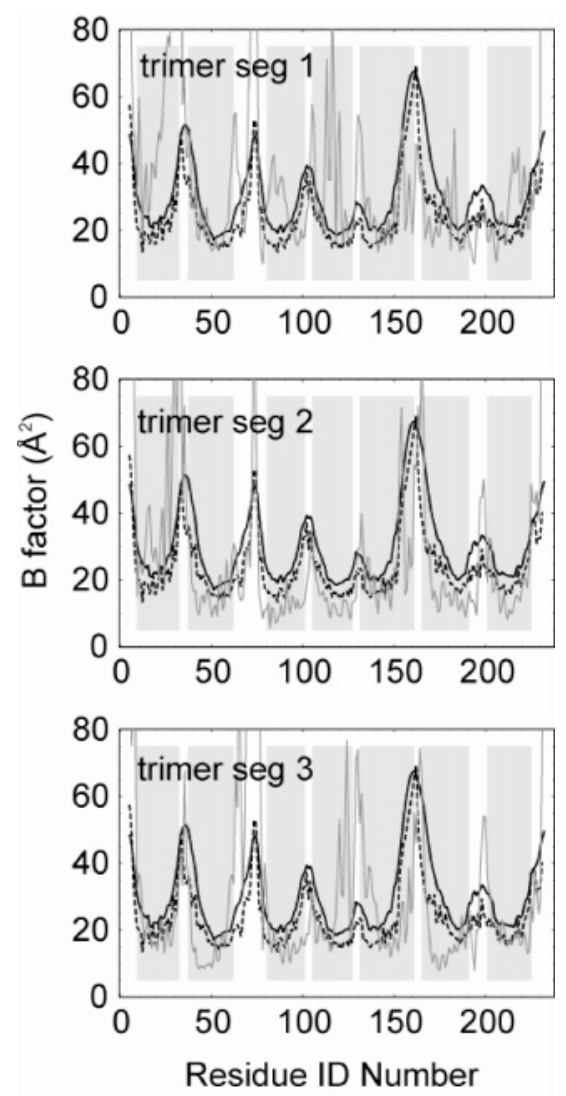

Figure 11. The B-factors of the $\mathrm{C}_{\alpha}$ atoms were calculated from their root-mean-square fluctuations. The solid gray line is from the HDGB simulation. The black solid (resolution $1.9 \AA$ ) $^{47}$ and dashed (resolution $1.55 \AA)^{22}$ lines are from the experiment. The region of the secondary structure elements (helices $A-G$ from the left) are indicated by the gray background.

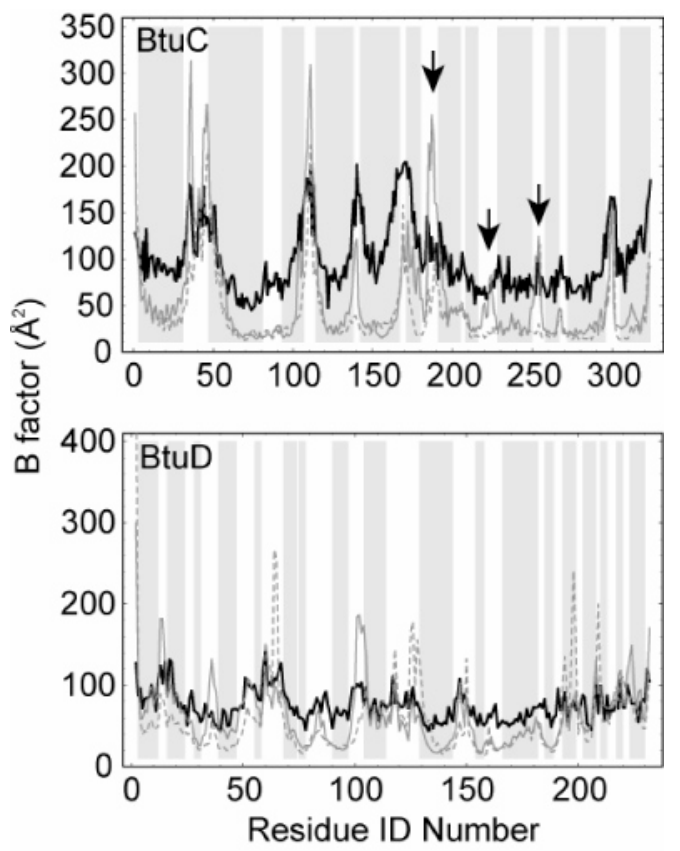

Figure 12. The B-factors of the $\mathrm{C}_{\alpha}$ atoms in the BtuCD aqueous solvent simulation were calculated from their root-mean-square fluctuations. The dotted gray line is for the calculated values, and the dark solid line is for the experiment. ${ }^{45}$ The region of the secondary structure elements are indicated by the gray background.

experimental values (Figure 12 top). Although $\mathrm{ABC}$ cassette subunits BtuD are in the aqueous environment, the B-factors 
of the BtuD in the aqueous solvent simulation (Figure 12 bottom) also show increased flexibility in some regions relative to results of the implicit membrane simulation.

\section{Discussion}

The data presented in this paper demonstrate that it is possible to carry out realistic simulations of integral membrane proteins with an implicit membrane environment. Both the thermodynamic stability and the dynamic properties were found to be in close agreement with explicit membrane simulations and experimental data for the bacteriorhodopsin and BtuCD system. The results are highly encouraging for simulations of other membrane-interacting biomolecules with an implicit membrane environment. An implicit representation of the environment may offer computational advantages for a single time step of molecular dynamics, but it is especially attractive in quantitative energetic analyses, because explicit sampling of the environmental degrees of freedom is not needed. Furthermore, an implicit representation of the lipid environment can be used to correct the insufficient level of polarization response in phospholipids with common fixed-charge force fields, ${ }^{55}$ which may otherwise require a polarizable force field. ${ }^{56-60}$ Nevertheless, the use of implicit descriptions of the environment is limited in cases where specific interactions with the environment are crucial. For example, proteins that interact with the phospholipid headgroup in a specific manner ${ }^{61}$ or the effect of cholesterol and polyunsaturated lipids in lipid-protein interactions ${ }^{62}$ could not be reproduced accurately with a purely implicit model. However, in some cases, it may be possible to include a limited number of solvent molecules while maintaining an implicit environment otherwise, for instance, by including only the solvent molecules in the interior of a channel protein explicitly. Membrane proteins with internal cavities or channels also pose another problem to the implicit membrane models developed so far (including the HDGB formalism): In many cases, water molecules occupy such cavities within the membrane bilayer plane, without, however, interacting directly with the phospholipids. To treat such solvent molecules implicitly, the current formalism would need to be extended to allow different dielectric environments, not just perpendicular to the membrane bilayer, but also along the bilayer. The HDGB model allows in principle such a modification, but the assignment of a local dielectric constant to atoms in a membrane protein with internal cavities is nontrivial and may need to be adjusted individually for a given protein. The current implicit membrane models also do not account for the effect of different ionic concentrations in the surrounding aqueous solvent, which is particularly relevant for ion channels. It is possible, however, to include the effect of low ionic concentrations at the Debye-Huckel level within the GB formalism. ${ }^{63}$

In most cases, the B-factors calculated from the HDGB simulations are somewhat lower than experimental data and comparable explicit solvent simulations. A good match of the dynamic properties in addition to the thermodynamic stability is important for ensuring that the implicit membrane simulations do not overstabilize the native state as a metastable configuration ${ }^{64,65}$ while allowing the possibility of carrying out quantitative kinetic analyses. The lower B-factors would suggest reduced dynamic fluctuations. However, it should also be considered that experimental structures at elevated resolutions tend to exhibit larger B-factors. In fact, the best agreement is found with the B-factors from the $1.55 \AA$ resolution $\mathrm{X}$-ray structure of the $\mathrm{bR}$ trimer, while the absolute B-factors deviate most for the BtuCD dimer, which was solved at a resolution of $3.2 \AA$.
The dynamic behavior with implicit solvent is modulated to a large extent through the choice of the friction coefficient in the Langevin heat bath. ${ }^{66}$ In this study, a value of $5 \mathrm{ps}^{-1}$ was used without any spatial dependence as a compromise between the high-friction aqueous solvent environment, where values of approximately $50 \mathrm{ps}^{-1}$ have been proposed, ${ }^{67}$ and the lowfriction hydrophobic tail region. From a recent comparison of GB simulations in aqueous solvent with friction coefficients of 5 and $50 \mathrm{ps}^{-1}$, it appears, however, that a value of $5 \mathrm{ps}^{-1}$ may be more realistic for aqueous solvent, so that even lower values would be appropriate for the membrane interior. In that case, the value of $5 \mathrm{ps}^{-1}$ used throughout the protein in the simulations described herein may suppress dynamic fluctuations in the membrane interior, as indicated by the calculated underestimated B-factors. Further tests are needed to establish more clearly which choice of friction coefficient is most appropriate in order to obtain the correct dynamic behavior with implicit descriptions of the environment.

As an indicator of the range of dynamic fluctuations, B-factors very similar to experimental data were found in the bR monomer simulation with the large electrostatic cutoff of $38 \AA$. In conjunction with the observation that electrostatic truncation may lead to serious artifacts in the anisotropic membrane environment, this finding would suggest that the choice of the electrostatic cutoff affects the quality of implicit membrane simulations even beyond cutoff distances of $18 \AA$ that are commonly considered acceptable with aqueous solvent. ${ }^{54}$ The need for a longer electrostatic cutoff in implicit membrane simulations may be understood from reduced electrostatic screening in low-dielectric environments. It is expected that similar issues would also arise in explicit membrane simulations. In fact, the occurrence of periodicity artifacts in membrane simulations with even relatively generous periodic box sizes ${ }^{55}$ also indicates the extended range of electrostatic interactions in low-dielectric membrane environments.

Simulations with an implicit aqueous solvent environment demonstrate larger differences in terms of structure and dynamics when compared to experimental data and explicit membrane simulations. However, despite overall qualitative differences, the baseline B-factors from the aqueous solvent simulations agree well with the experimental data, suggesting more flexible molecules than in the shorter-cutoff implicit membrane simulations. The difference may be related to the effect of electrostatic truncation, which is considered to be less significant in highdielectric aqueous solvent.

The present study represents first steps toward the simulation of integral membrane proteins with implicit membrane environments. However, further studies will be needed to fully understand the effect of the methodological aspects discussed above.

\section{Conclusion}

It was demonstrated that the HDGB implicit membrane model can be applied successfully in order to obtain stable and dynamically well-behaved trajectories of integral membrane proteins. Simulations of the bacteriorhodopsin monomer, trimer, and the BtuCD proteins remained close to the starting X-ray structure after 10,5 , and $5 \mathrm{~ns}$ of simulation time, respectively, while B-factors calculated from the simulations are in good agreement with experiment and explicit membrane simulations.

The effect of the electrostatic interaction cutoff distance was examined in the case of the $\mathrm{bR}$ monomer, where the protein was found to turn into a horizontal position with a certain cutoff distance. Furthermore, it was found that the agreement between 
calculated and experimental B-factors improves with essentially no electrostatic cutoff, suggesting that very long cutoff distances may ultimately be needed to obtain the most realistic simulations with implicit membrane environments.

\section{References and Notes}

(1) Voet, D.; Voet, J. G. Biochemistry, 3rd ed.; John Wiley and Sons: New York, 2004; Vol. 1.

(2) Wallin, E.; von Heijne, G. Protein Sci. 1998, 7, 1029.

(3) Boyd, D.; Schierle, C.; Beckwith, J. Protein Sci. 1998, 7, 201.

(4) Byrne, B.; Iwata, S. Curr. Opin. Struct. Biol. 2002, 12, 239.

(5) Bowie, J. U. Proc. Natl. Acad. Sci. U.S.A. 2004, 101, 3995.

(6) Leach, A. Molecular Modelling: Principles and Applications, 2nd ed.; Prentice Hall: New York, 2001.

(7) Schlick, T. Molecular Modeling and Simulation; Springer-Verlag:

New York, 2002.

(8) Warshel, A. Acc. Chem. Res. 2002, 35, 385.

(9) Brooks, C. L.; Karplus, M. J. Mol. Biol. 1989, 208, 159.

(10) Forrest, L. R.; Sansom, M. S. P. Curr. Opin. Struct. Biol. 2000 , 10,174 .

(11) Hansson, T.; Oostenbrink, C.; van Gunsteren, W. F. Curr. Opin. Struct. Biol. 2002, 12, 190 .

(12) Feig, M.; Brooks, C. L. Curr. Opin. Struct. Biol. 2004, 14, 217.

(13) Roux, B.; Simonson, T. Biophys. Chem. 1999, 78, 1.

(14) Schaefer, M.; Karplus, M. J. Phys. Chem. 1996, 100, 1578

(15) Jang, H.; Crozier, P. S.; Stevens, M. J.; Woolf, T. B. Biophys. J.

2004, 87, 129 .

(16) Bashford, D. An object-oriented programming suite for electrostatic effects in biological molecules. In Scientific Computing in Object-Oriented Parallel Environments; Ishikawa, Y., Oldehoeft, R. R., Reynders, J. V. W., Tholburn, M., Eds.; Springer-Verlag: New York, 1997; Vol. 1343, p 233.

(17) Gilson, M. K.; Honig, B. Proteins: Struct., Funct. Genet. 1988, 4,

(18) Holst, M.; Baker, N.; Wang, F. J. Comput. Chem. 2000, 21, 1319.

(19) Madura, J. D.; Briggs, J. M.; Wade, R. C.; Davis, M. E.; Luty, B.

A.; Ilin, A.; Antosiewicz, J.; Gilson, M. K.; Bagheri, B.; Scott, L. R.;

McCammon, J. A. Comput. Phys. Commun. 1995, 91, 57.

(20) Totrov, M.; Abagyan, R. Biopolymers 2001, 60, 124.

(21) Fogolari, F.; Brigo, A.; Molinari, H. Biophys. J. 2003, 85, 159.

(22) Luecke, H.; Schobert, B.; Richter, H. T.; Cartailler, J. P.; Lanyi, J.

K. J. Mol. Biol. 1999, 291, 899.

(23) Luo, R.; David, L.; Gilson, M. K. J. Comput. Chem. 2002, 23, 1244.

(24) Feig, M.; Onufriev, A.; Lee, M. S.; Im, W.; Case, D. A.; Brooks,

C. L. J. Comput. Chem. 2004, 25, 265.

(25) Still, W. C.; Tempczyk, A.; Hawley, R. C.; Hendrickson, T. J. Am. Chem. Soc. 1990, 112, 6127.

(26) Lee, M. S.; Feig, M.; Salsbury, F. R.; Brooks, C. L. J. Comput. Chem. 2003, 24, 1348.

(27) Stern, H. A.; Feller, S. E. J. Chem. Phys. 2003, 118, 3401.

(28) Tanizaki, S.; Feig, M. J. Chem. Phys. 2005.

(29) Im, W.; Feig, M.; Brooks, C. L. Biophys. J. 2003, 85, 2900.

(30) Spassov, V. Z.; Yan, L.; Szalma, S. J. Phys. Chem. B 2002, 106 , 8726.

(31) Qiu, D.; Shenkin, P. S.; Hollinger, F. P.; Still, W. C. J. Phys. Chem. A 1997, 101, 3005. 1691.

(33) Im, W.; Brooks, C. L. J. Mol. Biol. 2004, 337, 513. 6771.

(34) Im, W.; Brooks, C. L. Proc. Natl. Acad. Sci. U.S.A. 2005, 102
(35) Lazaridis, T. Proteins: Struct., Funct., Genet. 2003, 52, 176.

(36) Lazaridis, T. Proteins: Struct., Funct., Bioinf. 2005, 58, 518.

(37) Lazaridis, T.; Karplus, M. Proteins: Struct., Funct., Genet. 1999, $35,133$.

(38) Levy, R. M.; Zhang, L. Y.; Gallicchio, E.; Felts, A. K. J. Am. Chem. Soc. 2003, 125, 9523 .

(39) Feig, M.; Im, W.; Brooks, C. L. J. Chem. Phys. 2004, 120, 903.

(40) Marrink, S. J.; Berendsen, H. J. C. J. Phys. Chem. 1996, 100, 16729

(41) Brooks, B. R.; Bruccoleri, R. E.; Olafson, B. D.; States, D. J.; Swaminathan, S.; Karplus, M. J. Comput. Chem. 1983, 4, 187.

(42) MacKerell, A. D.; Bashford, D.; Bellott, M.; Dunbrack, R. L.; Evanseck, J. D.; Field, M. J.; Fischer, S.; Gao, J.; Guo, H.; Ha, S.; JosephMcCarthy, D.; Kuchnir, L.; Kuczera, K.; Lau, F. T. K.; Mattos, C.; Michnick, S.; Ngo, T.; Nguyen, D. T.; Prodhom, B.; Reiher, W. E.; Roux, B.; Schlenkrich, M.; Smith, J. C.; Stote, R.; Straub, J.; Watanabe, M. Wiorkiewicz-Kuczera, J.; Yin, D.; Karplus, M. J. Phys. Chem. B 1998 102,3586

(43) Oesterhelt, D. Curr. Opin. Struct. Biol. 1998, 8, 489.

(44) ABC Proteins: From Bacteria to Man; Holland, I. B., Cole, S. P. C., Kuchler, K., Higgins, C. F., Eds.; Academic Press: New York, 2002.

(45) Locher, K. P.; Lee, A. T.; Rees, D. C. Science 2002, 296, 1091 (46) Oloo, E. O.; Tieleman, D. P. J. Biol. Chem. 2004, 279, 45013.

(47) Belrhali, H.; Nollert, P.; Royant, A.; Menzel, C.; Rosenbusch, J. P.; Landau, E. M.; Pebay-Peyroula, E. Structure 1999, 7, 909.

(48) Gerwert, K.; Hess, B.; Soppa, J.; Oesterhelt, D. Proc. Natl. Acad. Sci. U.S.A. 1989, 86, 4943.

(49) Feig, M.; MacKerell, A. D.; Brooks, C. L. J. Phys. Chem. B $\mathbf{2 0 0 3 , ~}$ 107,2831

(50) Saam, J.; Tajkhorshid, E.; Hayashi, S.; Schulten, K. Biophys. J. 2002, 83, 3097.

(51) Ryckaert, J. P.; Ciccotti, G.; Berendsen, H. J. C. J. Comput. Phys. 1977, 23, 327.

(52) Aqvist, J. Comput. Chem. 1986, 10, 97.

(53) Kandt, C.; Schlitter, J.; Gerwert, K. Biophys. J. 2004, 86, 705.

(54) Norberg, J.; Nilsson, L. Biophys. J. 2000, 79, 1537.

(55) Allen, T. W.; Andersen, O. S.; Roux, B. Proc. Natl. Acad. Sci. U.S.A. 2004, 101, 117 .

(56) Anisimov, V. M.; Lamoureux, G.; Vorobyov, I. V.; Huang, N.; Roux, B.; MacKerell, A. D, Jr.. J. Chem. Theory Comput. 2005, 1, 153. (57) Duan, Y.; Wu, C.; Chowdhury, S.; Lee, M. C.; Xiong, G. M. Zhang, W.; Yang, R.; Cieplak, P.; Luo, R.; Lee, T.; Caldwell, J.; Wang, J. M.; Kollman, P. J. Comput. Chem. 2003, 24, 1999.

(58) Kaminski, G. A.; Stern, H. A.; Berne, B. J.; Friesner, R. A. J. Phys Chem. A 2004, 108, 621.

(59) Patel, S.; Brooks, C. L. J. Comput. Chem. 2004, 25, 1.

(60) Patel, S.; Mackerell, A. D.; Brooks, C. L. J. Comput. Chem. 2004 25,1504 .

(61) Malkowski, M. G.; Ginell, S. L.; Smith, W. L.; Garavito, R. M. Science 2000, 289, 1933.

(62) Pitman, M. C.; Grossfield, A.; Suits, F.; Feller, S. E. J. Am. Chem Soc. 2005, 127, 4576 .

(63) Bashford, D.; Case, D. A. Annu. Rev. Phys. Chem. 2000, 51, 129

(64) Fan, H.; Mark, A. E; Zhu, J.; Honig, B. Proc. Natl. Acad. Sci. U.S.A. 2005, 102, 6760

(65) Zhu, J.; Alexov, E.; Honig, B. J. Phys. Chem. B 2005, 109, 3008.

(66) Chandrasekhar, S. Rev. Mod. Phys. 1943, 15, 1.

(67) Widmalm, G.; Pastor, R. W. J. Chem. Soc., Faraday Trans. 1992 $88,1747$.

(68) Humphrey, W.; Dalke, A.; Schulten, K. J. Mol. Graphics 1996, 